\title{
Möglichst oft Resektabilität erreichen
}

FOLFOXIRI plus Bevacizumab erwies sich bei Patienten mit metastasiertem kolorektalem Karzinom (mCRC) wirksam im Sinn einer Konversion zu einer Resektabilität der Lebermetastasen, was sich unabhängig von ungünstigen klinischen und molekularen Faktoren günstig auf die Langzeitprognose auswirkte.

Die Autoren hatten die gepoolten Daten aus drei italienischen prospektiven Studien mit einer initialen Therapie mit FOLFOXIRI (5-Fluorouracil, Oxaliplatin und Irinotecan) plus Bevacizumab analysiert. In die aktuelle Auswertung wurden die 205 Patienten (37,9\%) der insgesamt 541 behandelten Patienten einbezogen, die eine nicht resektable, auf die Leber beschränkte Metastasierung aufwiesen. In $90 \%$ der Fälle waren die Lebermetastasen synchron, bei $79 \%$ bilöbär und $61 \%$ der Patienten wiesen vier und mehr Lebermetastasen auf. Bei $42 \%$ der Patienten waren Metastasen größer als 5 $\mathrm{cm}$, bei einem Viertel sechs und mehr Segmente betroffen.

36,1 \% der Patienten konnten aufgrund der intensivierten Upfront-Therapie R0oder R1-reseziert werden. R2-Rektionen wurden in 8,3\% der Fälle durchgeführt. Die Wahrscheinlichkeit für das Erreichen einer Resektionsfähigkeit durch die Therapie war erhöht bei weniger als sechs betroffenen Segmenten $(\mathrm{p}<0,001)$ und bei Erreichen der RECIST-Ansprechkriterien $(\mathrm{p}=0,019)$.

Patienten mit einer R0 - oder R1-Resektion wiesen mit 18,1 Monaten ein signifikant längeres progressionsfreies Überleben auf als die übrigen Patienten mit 10,7 Monaten (Hazard Ratio [HR] 0,48; p < 0,001). Das mediane Gesamtüberleben war fast verdoppelt (44,3 vs. 24,4 Mona-

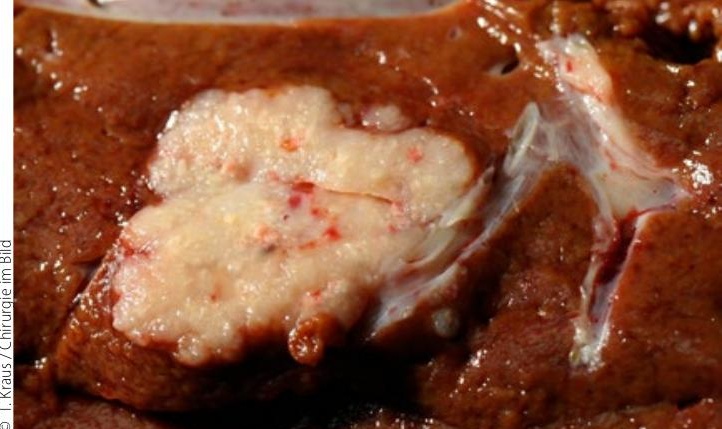

Lebermetastasen: Eine systemische Therapie kann die Chance auf Resektion steigern.

te; HR 0,32; $<<0,001)$. Die Unterschiede blieben auch in der multivariaten Analyse signifikant. Bei R0-Resektion lag das mediane progressionsfreie 5-JahresÜberleben bei $12 \%$, das mediane 5-Jahres-Gesamtüberleben bei $43 \%$.

Ungünstige Prognosefaktoren vor Therapiebeginn zeigten keine Assoziation mit dem postoperativen Therapieergebnis. Auch für RAS- und BRAF-Mutationen ließ sich keine solche Assoziation feststellen.

Friederike Klein

Cremolini C et al. Efficacy of FOLFOXIRI plus bevacizumab in liver-limited metastatic colorectal cancer: A pooled analysis of clinical studies by Gruppo Oncologico del Nord Ovest. Eur J Cancer. 2017;73:74-84.

\section{kurz notiert}

Immunisierung könnte den Tasmanischen Teufel retten

Der Tasmanische Teufel, auch Beutelteufel genannt, ist der größte noch lebende Raubbeutler. Er ist heute nur noch in Tasmanien zu finden. Sein Bestand ist jedoch durch einen tödlichen Gesichtstumor, die Devil Facial Tumor Disease (DFTD), bedroht. Dieser infektiöse Tumor der Schwann-Zellen wird durch Bisse übertragen. Die infizierten Tiere zeigen keine Antikörperoder Immunzellantwort auf die DFTD-Zellen, was dem Fehlen des Haupthistokompatibilitätskomplexes I (MHC-I) auf der Oberfläche der Tumorzellen zugeschrieben wird. Australische Wissenschaftler behandelten nun Tasmanische Teufel, die golfballgroße Tumoren aufwiesen, mit DFTD-Zellen, bei denen sie die MHC-I-Expression induziert hatten. Daraufhin schrumpften die Tumoren und waren nach drei Monaten vollständig verschwunden. Die Tumorregression war mit einer Antikörperantwort gegen DFTDZellen assoziiert. Bei keinem der fünf immunisierten Beutelteufel konnte sich der Tumor regenerieren. Somit konnte zum ersten Mal gezeigt werden, dass die Immuntherapie eine Tumorregression hervorrufen kann. Ermutigend sind die Ergebnisse auch deshalb, weil es möglich wurde, das Immunsystem so zu beeinflussen, dass es den Tumor erkannte und zerstörte [Tovar C et al. Sci Rep. 2017;7:43827]. Judith Neumaier

Pankreatische neuroendokrine Tumoren

\section{TKI hält neutraler Überprüfung stand}

Einige Zulassungsbehörden fordern neben der Einschätzung des Behandlungserfolgs durch den Untersucher auch die Beurteilung unabhängiger Gutachter. Im Fall der Therapie pankreatischer neuroendokriner Tumoren (PNET) mit dem Tyrosinkinaseinhibitor (TKI) Sunitinib ändert das am ursprünglichen Studienergebnis nichts.

In der placebokontrollierten Phase-IIIStudie mit Sunitinib (37,5 mg/Tag) lag das progressionsfreie Überleben nach der Einschätzung der Untersucher im TKIArm mehr als doppelt so hoch wie im Placeboarm (11,4 vs. 5,5 Monate; Hazard Ratio [HR] 0,42; p < 0,001). Die aktuelle Auswertung basiert auf der retrospektiven Begutachtung durch für Behandlungsarm und Nebenwirkungen verblindete Radiologen einer anderen Einrichtung sowie den finalen Ergebnissen zum Gesamtüberleben. Dabei wurde auch das Crossover von 69\% der Patienten vom Placebo-Arm zu einer Sunitinib-Therapie berücksichtigt. Ursprünglich wurden 171
Patienten mit fortgeschrittenem, gut differenziertem und progredientem PNET randomisiert, 86 hatten Sunitinib und 85 Placebo erhalten.

Die Auswertung der Gutachter ergab ein medianes progressionsfreies Überleben im Sunitinib-Arm von 12,6 Monaten und im Placebo-Arm von 5,8 Monaten. Damit lag die Hazard Ratio (HR) mit 0,32 $(\mathrm{p}=0,000015)$ noch unter der in der primären Auswertung. Fünf Jahre nach Ende der Studie betrug das mediane Gesamtüberleben der Patienten im SunitinibArm 38,6 Monate, im Placebo-Arm 29,1 Monate. Die HR war dabei knapp nicht signifikant $(0,73 ; p=0,094)$. Auch nach Berücksichtigung des Crossovers konnte der Vorteil im Gesamtüberleben für Sunitinib bestätigt werden.

Friederike Klein

Faivre S et al. Sunitinib in Pancreatic Neuroendocrine Tumors: Updated Progression-Free Survival and Final Overall Survival From a Phase III Randomized Study. Ann Oncol. 2016 Nov 10. [Epub ahead of print]. 